\title{
The 2q23.1 microdeletion syndrome: clinical and behavioural phenotype
}

\author{
Bregje WM van Bon ${ }^{1}$, David A Koolen ${ }^{1}$, Louise Brueton ${ }^{2}$, Dominic McMullan ${ }^{2}$, Klaske D Lichtenbelt ${ }^{3}$, \\ Lesley C Adès ${ }^{4}$, Gregory Peters ${ }^{4}$, Kate Gibson ${ }^{5}$, Francesca Novara ${ }^{6}$, Tiziano Pramparo ${ }^{6}$, \\ Bernardo Dalla Bernardina ${ }^{7}$, Leonardo Zoccante ${ }^{7}$, Umberto Balottin $^{7}$, Fausta Piazza ${ }^{7}$, Vanna Pecile ${ }^{8}$, \\ Paolo Gasparini ${ }^{9}$, Veronica Guerci ${ }^{9}$, Marleen Kets ${ }^{1}$, Rolph Pfundt ${ }^{1}$, Arjan P de Brouwer ${ }^{1}$, Joris A Veltman ${ }^{1}$, \\ Nicole de Leeuw ${ }^{1}$, Meredith Wilson ${ }^{10}$, Jayne Antony ${ }^{10}$, Santina Reitano ${ }^{11}$, Daniela Luciano ${ }^{12}$, Marco Fichera ${ }^{12}$, \\ Corrado Romano ${ }^{11}$, Han G Brunner ${ }^{1}$, Orsetta Zuffardi ${ }^{13,14}$ and Bert BA de Vries ${ }^{\star, 1}$
}

Six submicroscopic deletions comprising chromosome band 2q23.1 in patients with severe mental retardation (MR), short stature, microcephaly and epilepsy have been reported, suggesting that haploinsufficiency of one or more genes in the 2q23.1 region might be responsible for the common phenotypic features in these patients. In this study, we report the molecular and clinical characterisation of nine new 2q23.1 deletion patients and a clinical update on two previously reported patients. All patients were mentally retarded with pronounced speech delay and additional abnormalities including short stature, seizures, microcephaly and coarse facies. The majority of cases presented with stereotypic repetitive behaviour, a disturbed sleep pattern and a broad-based gait. These features led to the initial clinical impression of Angelman, Rett or Smith-Magenis syndromes in several patients. The overlapping 2q23.1 deletion region in all 15 patients comprises only one gene, namely, MBD5.

Interestingly, MBD5 is a member of the methyl CpG-binding domain protein family, which also comprises MECP2, mutated in Rett's syndrome. Another gene in the 2q23.1 region, EPC2, was deleted in 12 patients who had a broader phenotype than those with a deletion of MBD5 only. EPC2 is a member of the polycomb protein family, involved in heterochromatin formation and might be involved in causing MR. Patients with a 2q23.1 microdeletion present with a variable phenotype and the diagnosis should be considered in mentally retarded children with coarse facies, seizures, disturbed sleeping patterns and additional specific behavioural problems.

European Journal of Human Genetics (2010) 18, 163-170; doi:10.1038/ejhg.2009.152; published online 7 October 2009

Keywords: 2q23.1; Angelman; EPC2; MBD5; microdeletion; Rett

\section{INTRODUCTION}

Chromosomal aberrations are a well-known cause of mental retardation (MR). Several new microdeletion and duplication syndromes have been delineated ${ }^{1-5}$ since the introduction of high-resolution microarray techniques. Some of these give rise to clinically recognisable phenotypes such as 3q29 microdeletions, ${ }^{6} 15 \mathrm{q} 24$ microdeletions $^{7}$ and $17 \mathrm{q} 21.31$ deletions, ${ }^{3}$ whereas others, such as deletions in $15 \mathrm{q} 13.3$ or $16 \mathrm{p} 13.1,{ }^{8}$ are clinically more variable. Occasionally, a condition that was originally considered to be a contiguous gene deletion syndrome finally seems to be caused by the haploinsufficiency of a single gene, such as EHMT1 in the 9q34 deletion syndrome ${ }^{9}$ and $C B P$ in 16p13 deletions causing Rubinstein-Taybi syndrome..$^{10,11}$

In recent years, six submicroscopic deletions comprising chromosome band 2q23.1 have been reported. ${ }^{12-17}$ Unlike deletions mediated by a nonallelic homologous recombination, these deletions did not have common break points. Nevertheless, except for one case, all deletions did show a common region of overlap in the 2q23.1 region, including two candidate genes, $E P C 2$ and MBD5. It has been suggested that haploinsufficiency of one or both of these genes might be responsible for the common features in $2 \mathrm{q} 23.1$ microdeletion patients. ${ }^{13,14}$

Frequently reported, but rather nonspecific, features in these patients are severe MR, with pronounced speech impairment, short stature, microcephaly and seizures. However, because of the limited number of reported cases, it has been difficult to determine whether there is a recognizable and shared phenotype caused by haploinsufficiency of genes in the 2q23.1 region and further phenotypic studies in larger cohorts are required. ${ }^{14}$ In this study, we report on the molecular and clinical characterisation of nine new 2q23.1 microdeletion patients and on the clinical follow-up and further molecular

\footnotetext{
${ }^{1}$ Department of Human Genetics, Radboud University Nijmegen Medical Centre, Nijmegen, The Netherlands; ${ }^{2}$ Division of Medical and Molecular Genetics, University of Birmingham, Birmingham, UK; ${ }^{3}$ Department of Medical Genetics, University Medical Centre Utrecht, Utrecht, The Netherlands; ${ }^{4}$ Discipline of Paediatrics and Child Health, University of Sydney, Sydney, Australia; ${ }^{5}$ Genetic Health Queensland, Royal Brisbane and Women's Hospital, Queensland, Australia; ${ }^{6}$ Medical Genetics, University of Pavia, Pavia, Italy; ${ }^{7}$ Child Neuropsychiatry Unit, University of Verona, Policlinico G.B. Rossi, Verona, Italy; ${ }^{8}$ Cytogenetics Laboratory, IRCCS Burlo Garafano, Trieste, Italy; ${ }^{9}$ Medical Genetics, IRCCS Burlo Garofolo, University of Trieste, Trieste, Italy; ${ }^{10}$ Department of Clinical Genetics, Children's Hospital at Westmead, Sydney, Australia; ${ }^{11}$ Pediatrics and Medical Genetics, I.R.C.C.S. Associazione Oasi Maria Santissima, Troina, Italy; ${ }^{12}$ Laboratory of Genetic Diagnosis, I.R.C.C.S. Associazione Oasi Maria Santissima, Troina, Italy; ${ }^{13}$ Genetica Medica, Università di Pavia, Pavia, Italy; ${ }^{14}$ IRCCS Fondazione C. Mondino, Pavia, Italy

*Correspondence: Dr BBA de Vries, Department of Human Genetics, RUNMC, PO Box 9101, Nijmegen 6500 HB, The Netherlands. Tel: +31 243 613 946;

Fax: +31 243668 753; E-mail: B.devries@antrg.umcn.nl

Received 20 May 2009; revised 2 September 2009; accepted 2 September 2009; published online 7 October 2009
} 
characterisation of two patients who were previously reported only concisely. ${ }^{16,17}$ This report provides a review on a total of $152 \mathrm{q} 23.1$ microdeletion patients.

\section{MATERIALS AND METHODS}

\section{Patients: clinical characterisation}

In this study, 11 patients with a 2q23.1 microdeletion were included: nine new patients and two patients who were briefly reported previously. ${ }^{16,17}$ Except for patient 10 who had a de novo apparently balanced translocation $(46, \mathrm{XY}, \mathrm{t}(2 ; 7)(\mathrm{q} 31 \mathrm{q} 32)$, all patients showed a normal karyotype in G-banded chromosome studies. Clinical information was obtained from the respective physicians.

\section{DNA samples: molecular characterisation}

Array analysis. Submicroscopic deletions were detected by array analysis using different array platforms and no other clinically relevant CNVs were observed. All samples were tested according to the protocol provided by manufacturers and were analysed using the company's softwares. In patient 1 , $500 \mathrm{~K}$, and in patients 2 and 9,250K single-nucleotide polymorphism array was performed (Affymetrix Inc., Santa Clara, CA, USA) and analysed (CNAG 2.0). In patients 3, 5, 8a, 8b and 10, 244K oligonucleotide array CGH was performed (CGH Analytics, Agilent, Santa Clara, CA, USA). Patient 4 was analysed using a beadchip (HumanCNV370 Illumina, Illumina, San Diego, CA, USA; BeadStudio 1.0.2). In patient 6, BAC microarray $\mathrm{CGH}$ was performed (BlueGnome, BlueGnome, Cambridge, UK; Cytochip v 3.01). For patient 7 , $44 \mathrm{~K}$ oligomeric CGH array was used (Agilent). The position of array targets was mapped to the UCSC genome browser, Hg18 (http://genome.ucsc.edu/).

Targeted multiplex ligation probe amplification. To further characterise the proximal break point in patient 2, a set of nine synthetic multiplex ligation probe amplification (MLPA) probes in three genes (MBD5, EPC2 and KIF5C) was developed. The MLPA probes were designed in accordance with a protocol provided by MRC-Holland (www.MRC-holland.com). Probe hybridisation and analysis were carried out as previously described. ${ }^{18}$

Genomic real-time quantitative PCR analysis. SYBR Green-based genomic real-time quantitative PCR (gqPCR) analysis was performed on a 7500 Fast Real-Time PCR System using Power SYBR Green PCR Master Mix (both Applied Biosystems, Foster City, CA, USA) according to manufacturer's instructions. Primers were developed by the primer3 programme (http:// frodo.wi.mit.edu/cgibin/primer3/primer3_www.cgi) ${ }^{19}$ (Supplementary Table 1) and validated as described before. ${ }^{20}$ The melting curves of all PCR products showed a single PCR product. Copy numbers were measured relative to one reference gene: CFTR. DNA copy number differences between two samples were calculated by the $C_{\mathrm{t}}$ or $2^{\Delta \Delta} C_{\mathrm{t}}$ method. ${ }^{21}$

\section{RESULTS}

\section{Clinical data}

The clinical details of patients are described below and summarised in Table 1. Photographs of patients are shown in Figure 1.

Patient 1. This patient was born at term, with a weight of $1790 \mathrm{~g}$ (0.4th centile). She was admitted to the neonatal unit because of small size, feeding difficulties, hypothermia and mild respiratory distress. At the age of 1 year, it was apparent she had developmental delay. She sat independently at 8 months of age, bottom shuffled at 16 months and walked independently at $2^{1 / 2}$ years. On examination at the age of 2 years and 11 months, she had a broad-based gait and could not speak recognisable words. She was dysmorphic, with a broad forehead, mild brachycephaly, a round face, synophrys, a small nose, a depressed nasal bridge, downturned corners of the mouth, an everted full lower lip, short fifth fingers with clinodactyly and short toes. She was somewhat hirsute and had a fading strawberry naevus over her left scapula. She had an ejection systolic murmur because of mild pulmonary stenosis.
A cerebral MRI showed multiple high-signal foci in the subcortical white matter bilaterally, predominantly in the frontal lobes. Hearing and vision were normal. At the age of 5 years, she had reasonable understanding of simple language, but had limited speech. Her coordination was poor. She had sleep disturbances for which melatonin was prescribed. At the age of $71 / 2$ years, she had significant learning difficulties, social communication difficulties and repetitive behaviours, including teeth grinding, chewing materials and stereotypic hand movements. She was routine bound and obsessed with certain things. She was able to dress and feed herself. She walked independently with a stiff-legged gait. Weight was on the 50th centile and height and OFC $(49 \mathrm{~cm})$ on the 2 nd centile. She had absence spells, but a normal EEG. At the age of 10 years, behaviour had improved considerably after starting gluten and dairy-free diets. She was talkative, mainly single words and 2-3 word phrases. She tested negatively for Rett, Angelman syndromes and Smith-Magenis syndrome (SMS).

Patient 2. This girl was born at term weighing $3300 \mathrm{~g}$ (40th centile) with hip dysplasia and varus feet. Her development was delayed from birth, she walked at 2 years of age. She had myopia and astigmatism and suffered from respiratory and urinary tract infections. Cerebral MRI at the age of 2 years was normal. Seizures commenced at 3 years and 10 months. She had hyperphagia and chewed and licked objects. At the age of 7 years and 3 months, she had relative obesity, with a height of $111 \mathrm{~cm}$ ( $<3 \mathrm{rd}$ centile), an OFC of $49 \mathrm{~cm}$ (10th centile) and a weight of $26 \mathrm{~kg}$ ( $>97 \mathrm{th}$ centile). She had brachycephaly, a coarse face, a broad forehead, upslanting palpebral fissures, arched eyebrows, an open mouth with downturned corners and a broad chin. She had small puffy hands, fifth-finger clinodactyly and small fourth and fifth toes. Hyperphagia had decreased. She showed dyspraxia and stereotypic hand movements. She walked with a broad base, dressed herself, spoke in 2-3 word sentences and recognised pictograms. Under treatment, epilepsy was well controlled, with generally seven seizures a month. Behavioural problems worsened during times of increased seizure frequency, characterised by hyperactivity, restlessness, stubbornness and aggression. She had a high pain threshold and sleep disturbances, waking 6-8 times a night, treated with melatonin. Before array analysis, she was suspected to have SMS and tested negatively for Angelman syndrome.

Patient 3. She was born at term with a weight of $3710 \mathrm{~g}$ (80th centile), length of $51 \mathrm{~cm}$ (25th centile) and an OFC of $33.2 \mathrm{~cm}$ (50th centile). Facial dysmorphisms included a prominent forehead, hypertelorism and depressed nasal bridge. During the first year, she had periods of constipation. At 5 months, her head growth started to slow, and by the age of 18 months, she had true microcephaly. At that time, her milestones were moderately delayed and she showed stereotypic hand movements and mild limb hypertonia. She walked at 20 months of age. At the age of 3 years and 7 months, she presented with moderate psychomotor retardation and clearly impaired expressive language. She showed self-stimulatory behaviour. She had microcephaly (OFC $46 \mathrm{~cm},<3 \mathrm{rd}$ centile) and short stature (height $90 \mathrm{~cm}$, 3rd centile). Additional findings included bilateral occipital plagiocephaly, downturned corners of the mouth, a sacral dimple and hirsutism. Cerebral MRI, cardiological evaluation and abdominal ultrasound were normal. She never had seizures and had a normal sleeping pattern.

Patient 4. He was born at term by caesarean section because of placenta praevia, with an OFC of $35.5 \mathrm{~cm}$ (50th centile), a weight of $3150 \mathrm{~g}$ (40th centile) and a length of $48 \mathrm{~cm}$ (20th centile). Vision and 


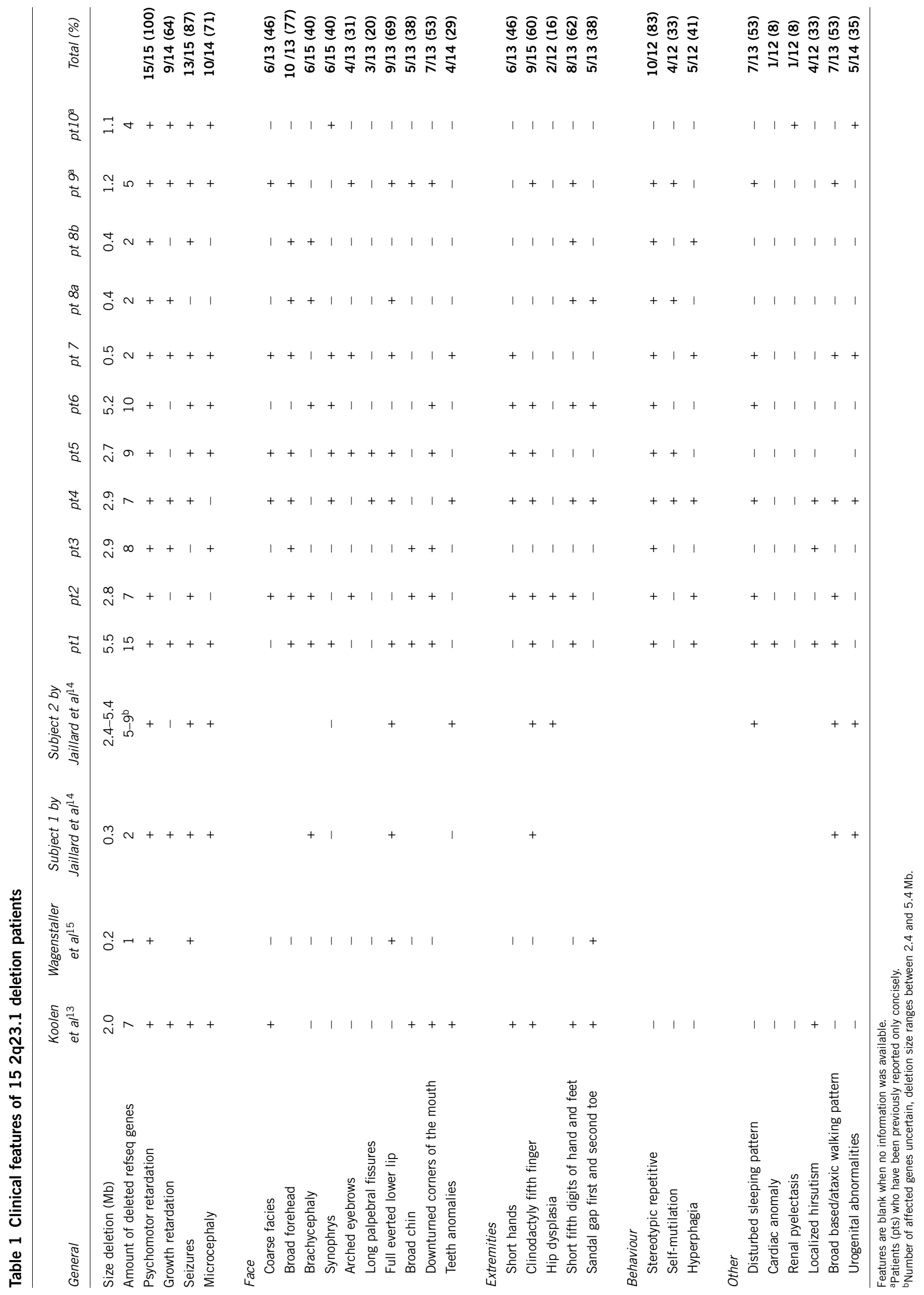


a
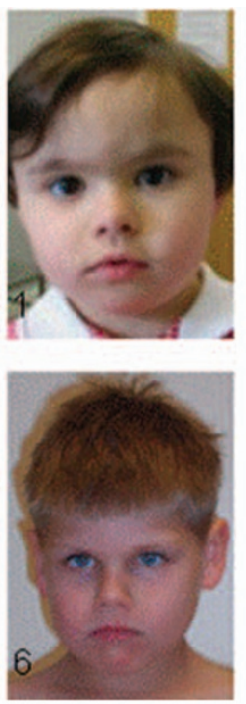
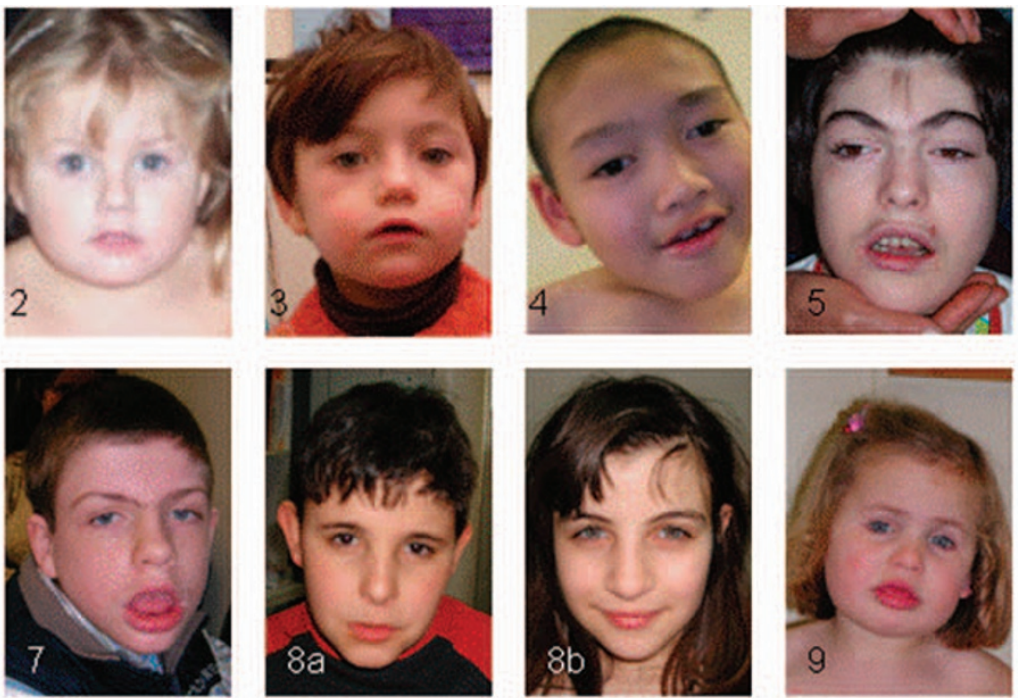

b chr2 (q22.3-q23.3)
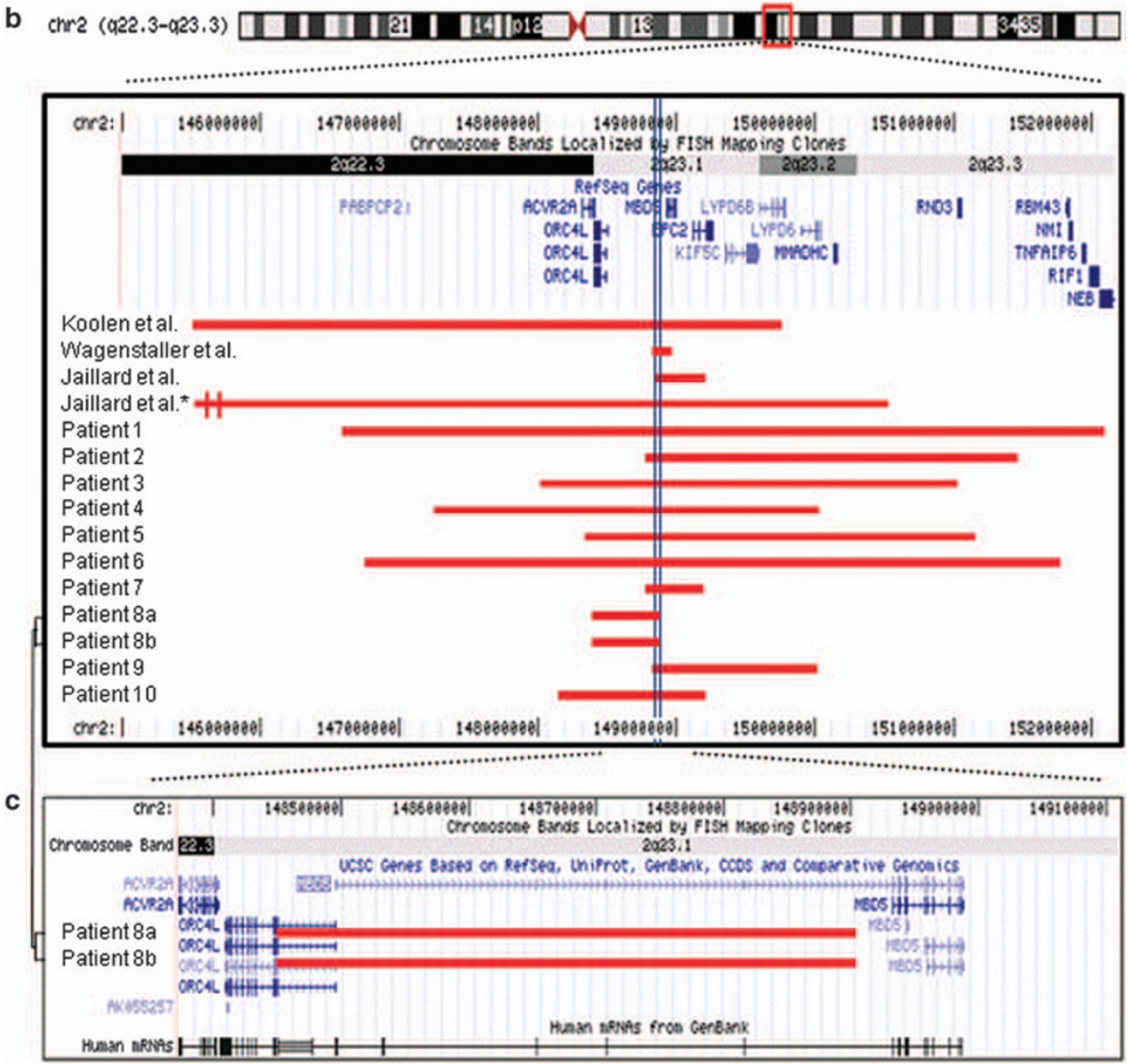

Figure 1 (a) Clinical photographs of all patients, except for patient 10. Note a broad chin in patients 1, 2, 3 and 9, downturned corners of the mouth in patients $1,2,3,5,6$ and 9, a full everted lower lip in patients $1,4,5,7,8 \mathrm{a}$ and 9 , a hypotonic face in patients 2, 3, 5, 7 and 9 and a coarse facial appearance in patients 2, 4, 5, 7 and 9. (b) Overview of all 2q23.1 deletions mapped in the UCSC genome browser Hg 18 (http://genome.ucsc.edu/). The overlapping region of all deletions comprises a minimal part of the untranslated region of the brain-expressed isoform of $M B D 5$. *The exact size of the deletion in case 2 of Jaillard et $a^{14}$ is unknown and varies between 2.4 and $5.4 \mathrm{Mb}$, with a maximum deletion between 145.1 and $150.6 \mathrm{Mb}$. (c) Deletions in sibpair $8 \mathrm{a}$ and $8 \mathrm{~b}$ include the non-coding region of the brain-expressed isoform of MBD5. The distal break point of deletions lies approximately $50 \mathrm{~kb}$ of the first exon of the regular isoform of MBD5. 
hearing were normal. Bilateral undescended testes were surgically repaired at 8 months of age. At 10 months of age, severe global developmental delay was noted. At the age of 17 months, an EEG showed startle-induced atonic seizures. Anti-convulsants were commenced. Cerebral MRI at the age of 6 years showed a thinning of the posterior corpus callosum. His milestones were delayed: he smiled at 4 months, rolled at 15 months, sat at 20 months, crawled at 4 years and walked independently at 5 years, with an ataxic wide-based stiff gait. $\mathrm{He}$ had ample vocalisation, but remained completely non-verbal. $\mathrm{He}$ could use one sign only by the age of 10 years. His behaviour was aggressive, self-mutilating and characterised by obsessions, a particular fascination with cars, incessant turning and tearing of magazine pages, frequent outbursts of laughter for no apparent reason, hand flapping, tooth grinding, chewing of objects and self-stimulation. He had excessive salivation, and chewed toys and the back of his hands. $\mathrm{He}$ had a high pain threshold, hyperacusis, was routine bound and had a clearly disturbed sleep pattern. At the age of 10 years and 8 months, OFC was $51.5 \mathrm{~cm}$ (16th percentile) and height $119 \mathrm{~cm}$ ( $<3 \mathrm{rd}$ centile). He had a coarse, dysmorphic face, with two posterior hair whorls over the scalp, a broad forehead, mid-facial hypoplasia, tongues of hair extending down from the pre-auricular regions to the cheeks, mild synophrys, long palpebral fissures, relative telecanthus (intercanthal distance $3.3 \mathrm{~cm}$; 75th-97th percentile), relative hypertelorism (interpupillary distance $6 \mathrm{~cm} ; 75 \mathrm{th}-97 \mathrm{th}$ percentile), protuberant ears with a slight uplifting of the left ear lobe, absence of an upper lateral incisor, prominent upper central incisors with a gap between them, full lips with an everted lower lip and prognathism. The midfinger, hand and foot lengths were in the $<3$ rd percentiles. He had foetal finger and toe pads, fifth-finger clinodactyly, short toes with a particular shortening of the first toes, a sandal gap, mirror clinodactyly of the second and third toes and clinodactyly of the fourth toes. Other features included hirsutism over the spine, generalised joint hypermobility, pes planus, truncal hypotonia and generalised hyper-reflexia. Before array analysis, he was suspected to have Angelman syndrome.

Patient 5. She was born at term with a weight of $4070 \mathrm{~g}$ (80th centile), a length of $52 \mathrm{~cm}$ ( 75 centile) and an OFC of $35 \mathrm{~cm}$ (50th centile). At 9 months of age, seizures commenced, and at 10 months, developmental delay was diagnosed. She had poor head control and could not hold objects. At the age of 26 months, she started to walk, but always needed support. Epilepsy continued during childhood, despite anti-convulsant therapy. She had absent acoustic reflexes. MECP2 analysis was negative. She had a normal sleep pattern. At 23 years of age, she had severe MR, no speech, gastroesophageal reflux and was unable to control her sphincters. She showed stereotypic movements and autistic features. She had a normal height and severe microcephaly ( $<3$ rd centile). Craniofacial dysmorphisms included a low frontal hairline, synophrys, arched eyebrows, long palpebral fissures, ptosis, low-set ears, a thin nasal bridge, short philtrum, a prominent upper lip, an everted lower lip, downturned corners of the mouth and a high and narrow palate. She had short stubby hands and feet. She died at the age of 26 years after several seizures.

Patient 6. This male was born at term with a weight of $4300 \mathrm{~g}$ (97th centile), a length of $51 \mathrm{~cm}$ (50th centile) and an OFC of $33.5 \mathrm{~cm}$ (10th centile). He had significant constipation and had faecal incontinence till the age of 6 years. Biopsy of bowel showed normal ganglion cells. At the age of 2 years, he had a febrile seizure and two prolonged absence episodes. He had developmental delay: he sat independently at 10 months, crawled at 17 months, walked at 21 months and said his first words at $2 \frac{1}{2}$ years. At the age of $3 \frac{1}{2}$ years, he had $>100$ signs and
15 words and could follow simple instructions. By the age of 5 years, he could use 4-5 word sentences and use a computer. EEG and MRI studies were normal. Since infancy, he had a sleep disorder characterised by apparent night terrors in the early part of sleep, waking in the early hours of the morning and sudden narcoleptic-like episodes later in the morning, during which he was difficult to wake. At 7 years of age, he had a height of $116 \mathrm{~cm}$ (3rd centile), a weight of $23 \mathrm{~kg}$ (25th centile) and an OFC of $47.5 \mathrm{~cm}$ ( $<3$ rd centile). He was brachycephalic and had soft, fine straight hair. He had a bitemporally narrowed forehead, heavy eyebrows, mild synophrys, deep set eyes, a short nose with slightly upturned nostrils, short philtrum, downturned corners of the mouth, a high palate and an open mouth appearance. In addition, he had simple and large ears with fleshy ear lobes. He had short fingers and toes, a sandal gap and a mild 2-3-toe cutaneous syndactly. He stood with lordotic posture and had three café au lait patches on his trunk. He had a deep and unusual mechanical quality to his voice. Angelman syndrome was excluded.

Patient 7. This boy was born at 36 weeks of gestation and had a weight of $2675 \mathrm{~g}$ (25th centile), a length of $43 \mathrm{~cm}$ (3rd centile) and an OFC of $31 \mathrm{~cm}$ (10th centile). He had feeding problems, intermittent tremors and neonatal jaundice requiring phototherapy. Subsequently, he was hypotonic with global developmental delay. He sat unsupported at 10 months, crawled at 16 months and started to cruise around furniture at the age of 20 months. He was an active, cheerful boy, who stood with everted feet. He had a square head shape, narrow arched eyebrows, synophrys, brachytelephalangy and foetal finger pads. He had a midline palatal groove, broad uvula and a large protruding tongue. His penis was small but testes and scrotum were normally developed. By the age of $2 \frac{1}{2} 2$ years, he had a short attention span, easy frustration, sleep disturbances and variable speech ability (up to five-word sentences). Cerebral CT scan showed mild cerebral atrophy and prominence of Sylvian fissures, and EEG was normal. At the age of 3 years, he developed tonic-clonic seizures. Except for mild hypermetropia, vision and hearing were normal. At 4 years of age, vesicoureteric reflux and some renal scarring were documented and seizure frequency markedly increased. He had a mixed seizure disorder with generalised tonic-clonic seizures, generalised tonic seizures, atonic seizures and absences. New EEGs showed multiregional epileptiform activity with some bilateral and generalised sharp wave activity. Cerebral MRI was normal. Until the age of 4 years, his OFC had been on the 5th centile, thereafter it decreased below the 3rd centile. At the age of 12 years, he had a severe epileptic encephalopathy, which was poorly controlled despite multiple anti-convulsants. He had episodes (up to $40 \mathrm{~h}$ ) of speech stereotypies, saying the same words repeatedly. He did not respond to questions, but had unprovoked verbalisations of short phrases. He could occasionally feed himself. He started finger picking when upset, but showed no selfmutilation. He had sleep disturbances, sometimes waking up hourly. He had a height of $132.5 \mathrm{~cm}$ ( $<3 \mathrm{rd}$ centile). At the age of 13 years, he developed episodes of marked daytime somnolence, during which he was difficult to rouse. His weight $(30 \mathrm{~kg})$ and $\mathrm{OFC}(50.4 \mathrm{~cm})$ were in the $<3$ rd centile. He had a hypotonic face, open mouth, a prominent tongue, thick lips with an everted lower lip, a high narrow palate, thick gums and widely spaced small teeth. He had marked synophrys with narrow eyebrows, a high nasal bridge and slightly upturned nares. $\mathrm{He}$ had shortened distal phalanges with thick square fingertips; his hand and mid-finger lengths were on the 3rd-25th percentile. He had mild kyphosis and walked with an ataxic gait.

FISH analysis for the SMS and methylation studies for Angelman syndrome were normal. 
Patient $8 a$. This male was born at term after a pregnancy characterised by threatened abortion. He has a sister with the same 2q23.1 aberration (patient $8 \mathrm{~b})$. At birth, he had a weight of $2230 \mathrm{~g}(<3 \mathrm{rd}$ centile), a length of $46 \mathrm{~cm}$ (20th centile), an OFC of $32 \mathrm{~cm}$ (20th centile) and presented with hypoglycaemia, tremors and cyanosis. During infancy, psychomotor, speech and growth delay became apparent. He walked at 23 months, spoke his first words at 30 months and was able to speak at 5 years of age. However, language was poor according to the Test for Reception Of Grammar (TROG test). Cerebral MRI at 4 years of age was normal. At 13 years, he presented with moderate MR, laxity of joints, hypermetropic astigmatism, muscle hypotonia and hypotrophy. An EMG showed mildly increased Motor Unit Potentials (MUP). He showed stereotypic movements such as hand flapping, including self-mutilation, for example, hand biting. He had a weight of $53 \mathrm{~kg}$ (95th centile), height of $138.5 \mathrm{~cm}$ $(<3$ rd centile) and an OFC of $53 \mathrm{~cm}$ (25th centile). Facial dysmorphisms included brachycephaly, a wide forehead, anteverted ears, a smooth philtrum, a thin upper lip and an everted full lower lip and a high palate. He had a dolichomegacolon, short first metacarpals, distal shortness of first digits, scoliosis, pes planovalgus and sandal gaps.

Patient $8 b$. This female, sister of patient $8 \mathrm{a}$, was born at term after an uneventful pregnancy. Her birth weight was $2900 \mathrm{~g}$ (20th centile). Four days after birth, she had a short period of cyanosis. She started to walk at 18 months and spoke her first words at 36 months. At the age of 10 years, anti-epileptic treatment was started after a seizure. At the age of 11 years, she had moderate MR, was able to communicate but used simple sentences and the TROG test showed understanding skills at a level of age 6 years. She had a weight of $42 \mathrm{~kg}$ (75th centile), height of $139.1 \mathrm{~cm}$ (25th centile) and an OFC of $51.4 \mathrm{~cm}$ (25th centile). She presented with stereotypic movements such as hand flapping and had hyperphagia, constantly searching for food. An EMG, because of muscle hypotonia and hypotrophy, showed mildly increased MUPs. In addition, she had hypermetropia, camptodactyly of thumbs and general laxity of other joints. Facial dysmorphisms included brachycephaly, a wide forehead, down slanted palpebral fissures and anteverted nostrils. She had scoliosis, pes planovalgus, lower limb asymmetry of $1.5 \mathrm{~cm}(\mathrm{~L}<\mathrm{R})$ and shortness of the fifth toes. Cerebral MRI at the age of 11 years showed ventricular asymmetry.

Patient 9. This female was born at term with a weight of $3750 \mathrm{~g}$ (75th centile). She was developmentally delayed from birth. She could sit at 1 year and started to walk at the age of 3 years. At the age of 10 months, seizures commenced. Cerebral MRI at the age of 1 year showed minimal abnormalities, with wide frontal ventricles and delayed myelinisation. An EEG at 13 months showed right temporal spikes and sharp waves, and right occipital slow waves. At the age of $4 \frac{1}{2}$ years, seizures were poorly controlled despite anti-convulsant therapy. She had severe MR, walked with a broad-based gait, had no speech and had feeding difficulties because of neuromotor problems and severe gastro-oesophageal reflux. At six years of age, her height was $107.5 \mathrm{~cm}$ ( $<3$ rd centile) and OFC $48 \mathrm{~cm}$ (10th centile). She had arched eyebrows, narrow ears, a full nasal tip, full lips, an everted lower lip, downturned corners of the mouth, volar finger pads, short fifth fingers, short great and fifth toes and mild 2-3-toe cutaneous syndactly. She had spastic and athetoid movements. When agitated, she displayed frequent picking of the eyes, periods of hyperpnoea, and would place her entire hands into her mouth. She required melatonin for her disturbed sleep pattern. She tested negatively for Angelman, Rett syndromes and SMS.
Patient 10. This boy was born after a pregnancy with a threatened miscarriage at 6 months of gestation. Maternal history revealed a spontaneous miscarriage at 3 months in an earlier pregnancy. At birth, he was cyanotic and required oxygen. His weight was $4460 \mathrm{~g}$ ( $>90$ th centile). During the initial days, he had left-sided seizures, and subsequently generalised seizures. The EEG revealed multifocal irritative abnormalities and his cerebral MRI was normal. An abdominal ultrasound showed mild bilateral renal pyelectasis and cryptorchidism. He started to walk at 20 months of age, with valgus position and external rotation of the feet.

At the age of $2 \frac{1}{2}$ years, he had sleep-related seizures, characterised by eye openings, upward deviation of the globes, hypertonia and perioral cyanosis. Otherwise, his sleep pattern was normal. He had delayed growth and psychomotor development with significant impairment of expressive language. At 6 years of age, he had an IQ of 36 on Griffith's Scale, verbal dyspraxia and poor communication. He showed serious aggressive behaviour towards others. His height was $104 \mathrm{~cm}$ (3rd centile) and OFC was $48 \mathrm{~cm}(<3$ rd centile). He had right occipital plagiocephaly with a prominent temporal region, synophrys, bilateral epicanthic folds, downslanting palpebral fissures, a saddle-like nasal root and micrognathia. Visual evoked potentials showed delayed cortical responses. Auditory evoked potentials were normal. Cerebral MRI showed pachygyria-like focal cortical abnormalities in the right temporal region. An EEG during partial sleep deprivation showed slowed bilateral fronto-central theta activity, especially on the right, and an additional superimposed fast activity. In the same areas, spike and spike-wave abnormalities were evident. The EEG pattern during sleep was nearly normal.

\section{Molecular findings}

The break points and sizes of deletions are summarised in Figure $1 \mathrm{~b}$ and Table 2. Except for patient 6, whose parents were unavailable, all patients showed a de novo deletion in $2 \mathrm{q} 23.1$ without other pathogenic losses or gains. The absence of a deletion in the parents of siblings $8 \mathrm{a}$ and $8 \mathrm{~b}$ is suggestive of gonadal mosaicism.

Single-nucleotide polymorphism array analysis of patient 2 could not reliably establish whether MBD5 was definitely within the deleted region. MLPA showed a half dosage ratio of all probes within $M B D 5$, EPC2 and KIF5C, indicating a deletion of these genes.

Quantitative PCR analysis was performed in siblings $8 \mathrm{a}$ and $8 \mathrm{~b}$ to confirm that only the brain-expressed isoform of MBD5 was deleted and not the more ubiquitously expressed regular isoform. The distal break point of the deletion indeed mapped within the untranslated region of the brain-expressed isoform of MBD5. On the basis of array

Table $2 \mathrm{Mb}$ positions of the $2 \mathrm{q} 23.1$ deletions determined in $\mathrm{Hg} 18$

\begin{tabular}{lccc}
\hline & Start position & End position & Size $(\mathrm{Mb})$ \\
\hline pt 1 & 146.6 & 152.2 & 5.5 \\
pt 2 & 148.7 & 151.5 & 2.8 \\
pt 3 & 148.1 & 151.0 & 2.9 \\
pt 4 & 147.2 & 150.1 & 2.9 \\
pt 5 & 148.4 & 151.1 & 2.7 \\
pt 6 & 146.7 & 151.8 & 5.2 \\
pt 7 & 148.7 & 149.2 & 0.5 \\
pt 8a & 148.5 & 148.9 & 0.4 \\
pt 8b & 148.5 & 148.9 & 0.4 \\
pt 9 & 148.8 & 150.0 & 1.2 \\
pt 10 & 148.1 & 149.2 & 1.1 \\
\hline
\end{tabular}

Abbreviations: pt, patients. 
analysis and $\mathrm{qPCR}$ data, the break point is located approximately $50 \mathrm{~kb}$ proximal to the first exon of the regular MBD5 isoform (Figure 1c and supplementary Figure 1).

\section{DISCUSSION}

In this clinical report, we present nine new cases with a deletion comprising the 2q23.1 region, and an extended clinical follow-up in two patients who have been previously reported. ${ }^{16,17}$ Including the available literature cases, ${ }^{13,14}$ we provide a review of 15 cases.

In addition to a moderate or severe MR, the majority ( $>50 \%)$ of patients with a 2q23.1 microdeletion had seizures, short stature, microcephaly, a broad forehead, downturned corners of the mouth, a full everted lower lip, short fifth fingers with clinodactyly, sleep disturbance, a broad-based ataxic walking pattern and stereotypic repetitive behaviour. Other common (20-50\%) features were a coarse face, facial dysmorphisms (such as brachycephaly, synophrys, arched eyebrows, long palpebral fissures, broad chin and teeth anomalies), short hands, sandal gap, hirsutism, self-mutilation and hyperphagia. Genital abnormalities were noted in five patients (35\%): a small penis (subject 1 by Jaillard et $a l^{14}$ and patient 7), hypoplastic genitalia (subject 2 by Jaillard et $a l^{14}$ ) and cryptorchidism (patients 4 and 5). Some unique congenital abnormalites were seen in single or two patients such as hip dysplasia, renal pyelectasis and cardiac defects. In contrast to previous reports that suggested the possibility of a recognisable facial phenotype, ${ }^{13,14}$ we were unable to delineate a distinctive pattern of facial dysmorphisms. The combination of coarse facial features and a specific behavioural pattern, however, point clinically towards a 2q23.1 microdeletion. In fact, many patients were tested for Angelman $(n=8)$, Rett $(n=4)$ and/or Smith-Magenis $(n=5)$ syndromes, which are all well known for their specific behavioural phenotype.

Typical behavioural characteristics of Angelman's syndrome are paroxysms of easily provoked laughter and ataxic gait. ${ }^{22}$ Some patients (20\%) with a 2q23.1 deletion showed similar behaviour with outbursts of inappropriate laughter. Most patients (53\%) had motor developmental delay, poor coordination and a broad-based/ataxic gait. In addition, Angelman's syndrome might also be considered because of limited or absent speech, sleep disturbance and seizures. Speech was variable in 2q23.1 microdeletion patients and ranged from non-verbal sounds to short sentences. Most patients understood simple language and/or pictograms, but had little or no effective communication. Severe sleep disturbance was present in seven cases (53\%), and was often treated with melatonin. Epilepsy was present in 13 cases $(87 \%)$. The EEGs in 2q23.1 microdeletion patients did not, however, show the classical Angelman's syndrome pattern. ${ }^{14}$

Stereotypic repetitive behaviour $(83 \%)$ and the presence of seizures $(87 \%)$ in $2 \mathrm{q} 23.1$ deletion patients led to the suspicion of Rett's syndrome in four patients, but all tested normal for MECP2. Stereotypic behaviour consisted of repetitive hand movements, teeth grinding and the chewing of hands and materials. Onset of seizures was unpredictable, varying from the first days of life to 10 years of age.

Finally, five patients were negatively tested for the SMS because of the suggestive behaviours of self-mutilation, stereotypies and aggression. ${ }^{23}$ In addition, almost all patients with SMS have sleep disturbance and language delay, ${ }^{23}$ as could also be observed in $2 \mathrm{q} 23.1$ deletion patients.

Deletion sizes varied from $250 \mathrm{~kb}$, including two genes, to $5.5 \mathrm{Mb}$ comprising 15 genes. The overlapping deleted 2q23.1 region comprised a minimal part of the untranslated region of $M B D 5$, suggesting that deletions of this region cause MR, stereotypic behaviour and seizures. MBD5 is a member of the methyl-CpG-binding domain
(MBD) protein family. Methylation at $\mathrm{CpG}$ dinucleotides in genomic DNA is an essential epigenetic mechanism of gene expression control in vertebrates. Proteins with an MBD can bind to methylated CpGs, thereby mediating effects of DNA methylation. ${ }^{24}$ Another well-known member of this group is MECP2, mutated in Rett's syndrome. ${ }^{24}$ Interestingly, several 2q23.1 deletion patients were tested for Rett's syndrome. Convincing evidence that haploinsufficiency of MBD5 leads to MR was reported by Wagenstaller et al, ${ }^{15}$ who described a mentally retarded male with seizures, who had an intragenic MBD5 deletion extending from the last exon in the non-coding region until exon 7 of the regular isoform. They also reported missense variants in MBD5 in four MR patients, of which the clinical implications remain unknown. In this study, we report a mentally retarded sibpair with a deletion of the non-coding region of $M B D 5$, adding additional evidence to MBD5 as an MR gene. However, except for a full everted lower lip, both the patient reported by Wagenstaller et $a^{15}$ and sibpair 8 did not show other characteristics such as a coarse face, microcephaly, genital abnormalities, a broad-based/ataxic gait and a disturbed sleep pattern, features frequently noted in the remainder of the 2q23.1 deletion cohort. This suggests that the full range of clinical features of 2q23.1 deletion patients is because of contiguous gene deletions, rather than of $M B D 5$ alone. Except for the patient reported by Wagenstaller et $a l^{15}$ and sibpair $8 \mathrm{a}$ and $8 \mathrm{~b}$, all patients had a deletion also including the EPC2 gene. Interestingly, two patients (case 1 Jaillard et al ${ }^{14}$ and patient 7) had a deletion including MBD5 and EPC2 only. Speech and motor developments were more impaired than in sibpair 8. In addition, both had microcephaly, a broad-based/ataxic walking pattern and urogenital abnormalities. EPC2 is a member of the polycomb protein family, involved in heterochromatin formation. ${ }^{25}$ This family of highly conserved proteins seems to be directly involved in essential epigenetic events governing both transcriptional activation and repression. ${ }^{26}$ Other examples of genes associated with $\mathrm{MR}$ and epigenetic regulation are JARID1C, MECP2, CDKL5 and EHMT1. These genes belong to a small group of known MR genes, which have a direct role in the modulation of chromatin structures. ${ }^{27}$ On the basis of our findings, EPC2 might be included in this category of MR genes leading to defective chromatin formation as the underlying mechanism, although definite proof on single EPC2 deletions/ mutations needs to be awaited.

In conclusion, the clinical phenotype in 2 q23.1 deletion patients is variable. A coarse facies in a mentally retarded child with seizures, sleep disturbances and rather specific behavioural problems will point towards this deletion. Deletions comprising both MBD5 and EPC2 seem to cause a more extensive phenotype than deletions of MBD5 alone. Moreover, a 2q23.1 microdeletion should be considered in any child with a differential diagnosis of the Angelman, Rett syndromes or SMS.

\section{ACKNOWLEDGEMENTS}

We thank all parents and children who participated in this study. This work was supported by grants from the European commission: AnEUploidy project (LSHG-CT-2006-037627) under FP6, the Netherlands Organisation for Health Research and Development (ZonMW 917-86-319 to B.B.A.d.V), Hersenstichting Nederland (B.B.A.d.V.), the Telethon project GGP05177 (TP) and the CARIPLO Foundation (project 2007.5197 to OZ).

1 Mefford HC, Sharp AJ, Baker C et al: Recurrent rearrangements of chromosome 1q21.1 and variable pediatric phenotypes. N Engl J Med 2008; 359: 1685-1699.

2 Gajecka M, Mackay KL, Shaffer LG: Monosomy 1 p36 deletion syndrome. Am J Med Genet C Semin Med Genet 2007; 145C: 346-356. 
3 Koolen DA, Sharp AJ, Hurst JA et al: Clinical and molecular delineation of the 17q21.31 microdeletion syndrome. J Med Genet 2008; 45: 710-720.

4 Ben-Shachar S, Ou Z, Shaw CA et al: 22q11.2 distal deletion: a recurrent genomic disorder distinct from DiGeorge syndrome and velocardiofacial syndrome. Am J Hum Genet 2008; 82: 214-221.

5 Ballif BC, Hornor SA, Jenkins E et al: Discovery of a previously unrecognized microdeletion syndrome of 16p11.2-p12.2. Nat Genet 2007; 39: 1071-1073.

6 Ballif BC, Theisen A, Coppinger J et al: Expanding the clinical phenotype of the 3q29 microdeletion syndrome and characterization of the reciprocal microduplication. Mol Cytogenet 2008; 1 : 8.

7 Sharp AJ, Selzer RR, Veltman JA et al: Characterization of a recurrent 15q24 microdeletion syndrome. Hum Mol Genet 2007; 16: 567-572.

8 Ullmann R, Turner G, Kirchhoff M et al: Array CGH identifies reciprocal 16p13.1 duplications and deletions that predispose to autism and/or mental retardation. Hum Mutat 2007; 28: 674-682.

9 Kleefstra T, Brunner HG, Amiel J et al: Loss-of-function mutations in euchromatin histone methyl transferase 1 (EHMT1) cause the 9q34 subtelomeric deletion syndrome. Am J Hum Genet 2006; 79: 370-377.

10 Petrij F, Giles RH, Dauwerse HG et al: Rubinstein-Taybi syndrome caused by mutations in the transcriptional co-activator CBP. Nature 1995; 376: 348-351.

11 Breuning MH, Dauwerse HG, Fugazza G et al: Rubinstein-Taybi syndrome caused by submicroscopic deletions within 16p13.3. Am J Hum Genet 1993; 52: 249-254.

12 Vissers LE, de Vries BB, Osoegawa $\mathrm{K}$ et al: Array-based comparative genomic hybridization for the genomewide detection of submicroscopic chromosomal abnormalities. Am J Hum Genet 2003; 73: 1261-1270.

13 Koolen DA, Vissers LE, Nillesen W et al: A novel microdeletion, del(2)(q22.3q23.3) in a mentally retarded patient, detected by array-based comparative genomic hybridization. Clin Genet 2004; 65: 429-432.

14 Jaillard S, Dubourg C, Gérard-Blanluet M et al: 2q23.1 microdeletion identified by array-CGH: an emerging phenotype with Angelman-like features? J Med Genet 2008 (epub ahead of print).
15 Wagenstaller J, Spranger S, Lorenz-Depiereux B et al: Copy-number variations measured by single-nucleotide-polymorphism oligonucleotide arrays in patients with mental retardation. Am J Hum Genet 2007; 81: 768-779.

16 De Gregori M, Ciccone R, Magini $\mathrm{P}$ et al: Cryptic deletions are a common finding in 'balanced' reciprocal and complex chromosome rearrangements: a study of 59 patients. J Med Genet 2007; 44: 750-762.

17 de Vries BB, Pfundt R, Leisink $M$ et al: Diagnostic genome profiling in mental retardation. Am J Hum Genet 2005; 77: 606-616.

18 Schouten JP, McElgunn CJ, Waaijer R, Zwijnenburg D, Diepvens F, Pals G: Relative quantification of 40 nucleic acid sequences by multiplex ligation-dependent probe amplification. Nucleic Acids Res 2002; 30: e57.

19 Rozen S, Skaletsky H: Primer3 on the WWW for general users and for biologist programmers. Methods Mol Biol 2000; 132: 365-386.

20 Marcelis CL, Hol FA, Graham GE et al: Genotype-phenotype correlations in MYCNrelated Feingold syndrome. Hum Mutat 2008; 29: 1125-1132.

21 Livak KJ, Schmittgen TD: Analysis of relative gene expression data using real-time quantitative PCR and the 2(-Delta Delta C(T)) Method. Methods 2001; 25: 402-408.

22 Clayton-Smith J, Laan L: Angelman syndrome: a review of the clinical and genetic aspects. J Med Genet 2003; 40: 87-95.

23 Gropman AL, Duncan WC, Smith AC: Neurologic and developmental features of the Smith-Magenis syndrome (del 17p11.2). Pediatr Neurol 2006; 34: 337-350.

24 Roloff TC, Ropers HH, Nuber UA: Comparative study of methyl-CpG-binding domain proteins. BMC Genomics 2003; 4: 1.

25 Doyon Y, Selleck W, Lane WS, Tan S, Cote J: Structural and functional conservation of the NuA4 histone acetyltransferase complex from yeast to humans. Mol Cell Biol 2004; 24: 1884-1896.

26 Perry J: The Epc-N domain: a predicted protein-protein interaction domain found in select chromatin associated proteins. BMC Genomics 2006; 7: 6 .

27 Kramer JM, van $\mathrm{BH}$ : Genetic and epigenetic defects in mental retardation. Int J Biochem Cell Biol 2009; 41: 96-107.

\title{
Corrigendum to: The 2q23.1 microdeletion syndrome: clinical and behavioural phenotype
}

\author{
Bregje WM van Bon, David A Koolen, Louise Brueton, Dominic McMullan, Klaske D Lichtenbelt, \\ Lesley C Adès, Gregory Peters, Kate Gibson, Francesca Novara, Tiziano Pramparo, Bernardo Dalla Bernardina, \\ Leonardo Zoccante, Umberto Balottin, Fausta Piazza, Vanna Pecile, Paolo Gasparini, Veronica Guerci, \\ Marleen Kets, Rolph Pfundt, Arjan P de Brouwer, Joris A Veltman, Nicole de Leeuw, Meredith Wilson, \\ Jayne Antony, Santina Reitano, Daniela Luciano, Marco Fichera, Corrado Romano, Han G Brunner, \\ Orsetta Zuffardi and Bert BA de Vries
}

European Journal of Human Genetics (2010) 18, 170; doi:10.1038/ejhg.2009.200

Correction to: European Journal of Human Genetics advance online publication 7 October 2009; doi: 10.1038/ejhg.2009.152

Since the publication of the above paper, the authors noticed a co-author was missing from the authors' list: Dr Susan Moloney, and her affiliation is MBBS FRACP, Department of Paediatrics, Gold Coast Health Service District, Southport, Gold Coast, Queensland, Australia. The complete authors' list is reproduced below:

Bregje WM van Bon, David A Koolen, Louise Brueton, Dominic McMullan, Klaske D Lichtenbelt, Lesley C Adès, Gregory Peters,
Kate Gibson, Susan Moloney, Francesca Novara, Tiziano Pramparo, Bernardo Dalla Bernardina, Leonardo Zoccante, Umberto Balottin, Fausta Piazza, Vanna Pecile, Paolo Gasparini, Veronica Guerci, Marleen Kets, Rolph Pfundt, Arjan P de Brouwer, Joris A Veltman, Nicole de Leeuw, Meredith Wilson, Jayne Antony, Santina Reitano, Daniela Luciano, Marco Fichera, Corrado Romano, Han G Brunner, Orsetta Zuffardi and Bert BA de Vries

The authors would like to apologise for this mistake. 